\title{
Teaching Reform of Web Page Design Based on Computer Vision
}

\author{
TianLei $^{1}$, MaYin $^{1}$, LiuZhigang $^{2, a}$ \\ ${ }^{1}$ Department of Electronic Information Engineering ,Henan Polytechnic Institute, \\ Nanyang 541004, China; \\ ${ }^{2}$ Nanchang Institute of Science \&Technology,Nanchang 330108,China \\ aliuzhigang_777@qq.com
}

Keywords: computer vision;web page design;teaching reform;aesthetic forms;target audience;color collocation

\begin{abstract}
Teaching Web page designed to co-ordinate the planning, the overall style of uniform. In order to make the site each page there is a whole, we should around a visual center to build an overall visual convey teaching information, Designers to comprehensively consider the various visual elements, especially the page in prominent characteristics at the same time pay attention to the harmony with the whole. From the perspective of computer vision and analyzed in some, For each specific page, based on the principle of overall design, the assurance of primary and secondary, the arrangement of colors, text, graphics processing and page layout discretion should be overall planning, This paper discusses the general requirements of computer vision design in teachingwebsites and the specific design method.
\end{abstract}

\section{Introduction}

Essence of teaching website is to transfer teaching information, providing teaching services, If teaching site teaching information only stay in a simple list level, the teaching content will lose their appeal, so that teaching cannot be effectively disseminate information. computer vision design in teaching websites is the art of a two-dimensional space, Based on principles of teaching information effectively convey, on the basis of network and multimedia technology, integrating teaching spirit and aesthetic value at an organic whole, To realize the teaching information and visual elements integration, comprehensive coordination, integrity and systemic.

\section{The Design of computer vision}

The computer vision design is based on expression of information and communication transmission as the research object, for the purpose of accurate expression and efficient information communication, to visual design and processing of information, so as to meet the needs of the people communication. computer vision design of the main function is to convey information, it is different from abstract concepts express by language, but through visual symbols to convey. The process of computer vision is used between people "see" form of communication, it is through the visual symbolic language expression and transmit. With the deepening of the information age, computer vision design will be completed by a single media to multimedia combination process, shows people various personalized needs, add vitality of science and technology to the human development of the information age. computer vision design will be through a variety of content and form of the attractive penetrated into every corner of life, in the process of information play a bigger role.

\section{Computer vision Design Principles and Methods in Teaching Website}

With the coming of network era, the teaching website as a new form of teaching resources will become a hotspot of research on the future; the computer vision design in teaching websites also should follow certain ideas. Website design should take the education as the starting point ,for visitors to provide the most comfortable visual environment, and improve the effect of teaching information effectively. 


\section{Computer vision Design Principles}

Teaching website of computer vision design is the integration of technology and art, the unity of content and form. Web page is text, graphics; color these the clever combination of multimedia elements, to provide visitors with the most direct visual feeling, Page can attract visitors, make the teaching better information communication is the key to the teaching website design. Designers in the computer vision design concept are applied to the teaching website should grasp the following three principles:

\section{Layout Reasonable}

Page layout is the designer of all design elements for the reasonable arrangement, it is not follow one's inclinations dreamt up, but designers cleverly use fabric and layout principle, Follow the rules of certain visual, will have different size, different shape, different color, and texture of design elements, give person with a unique visual art feeling, Create the aesthetic forms of information, In the design page, should put the most important content in the screen to the left or top area. The focus content of the rest in page can through the text arrangement, the collocation of color or through other means, the visitor's attention to it.

\section{Coloring Accurate}

Color and hue is the most emotional, the most appealing visual elements, color is the most cultural elements. Main color selection and design not only reflects the topic of the page, and dominate the entire page design. Color design should be based on the content of the needs and different browsers color, choose the color psychological characteristics and deal with the color contrast and harmony. Different disciplines, different content of the same subject to the requirement of color is not the same, should be based on the performance of the content need to select and match.

\section{Harmonious and unified}

Coordinated unification is the most basic aesthetic standards of web page design. Color and rhythm are factors to consider in the design of the page, but how to put these elements together, to make them coordinate with each other, to serve the theme of the website, this is the most important. Ultimately, all the elements on the page, will ultimately be unified by the themes expressed. That is to say, the reasonable expression of layout, color, rhythm and must obey the theme as the premise, between various visual elements to match each other, coordinate with each other.

\section{computer vision Design Method in the Teaching Website}

\section{Clear Target Audience}

Teaching Web page visual design success is directly related to the viewer's first impression of the site is related to the viewer of the site an overall rating. When making the page design designers must clarify the main users of web site is what kind of people, and the users are classified, analyzed what they want from the site, They have what kind of aesthetic tendency, have what kind of Internet psychology, etc. For different user groups, the need for different design, page design and the performance theme changes with the change of the main users.

\section{Clear Site Purpose}

A website to the visitor what to propaganda, what to introduce, what to promote, must get clearly show in the page design, That is to say, the design should achieve the purpose of this page, your visitors through the web page to browse, basically can to grasp and understanding of website content and characteristics. 


\section{The computer vision Design in Teaching Websites}

Teaching website page is mainly consists of text, graphics, color, format, such as visual elements, these elements not only convey information, but also express emotions, cause emotional feelings rich psychological activity. In order to make the web page of obtaining maximum visual information ability, web design must adapt to the visual psychological and physiological characteristics. This article mainly from the text, color, graphics, layout format four aspects is to carry on the design.

\section{The Design of Text}

In teaching websites, the text is the core of the page, also is the most direct way of computer vision, we gain knowledge mainly through the text, and site is mainly through the text to improve the thought. Text itself has much potential expression, such as different font, font size; layout will produce different psychological induction. Good use of these factors, can make the text appear to be very good to convey the designer of the atmosphere, make the text has overall demand effect, give a person with clear visual perception, to deepen the visitor's impression. Different fonts have different performance, some font humor, serious, some font display some font display soft, some font display power, these are the font of emotional expression. A good web design without the choice of font, if the font in the design no good choice, then may affect the overall effect of a web page. The page size proportion, is an important aesthetics principle of visual aesthetic, directly affects the visitor's learning efficiency. The choice of font size according to the functional needs, the larger the word to the audience the visual impact of the strong, the longer the time of memory, The fine print is appear delicate and exquisite attractive, small precision higher and higher, and the word is easy to form, the stronger the integrity, But the word is too small, too much content, and easy to make the page into multiple centers, cause page fuzzy, lack of sense of beauty, influence visitors visual aesthetic space. Different font size, font will show different rendering force, as shown in Figure 1.

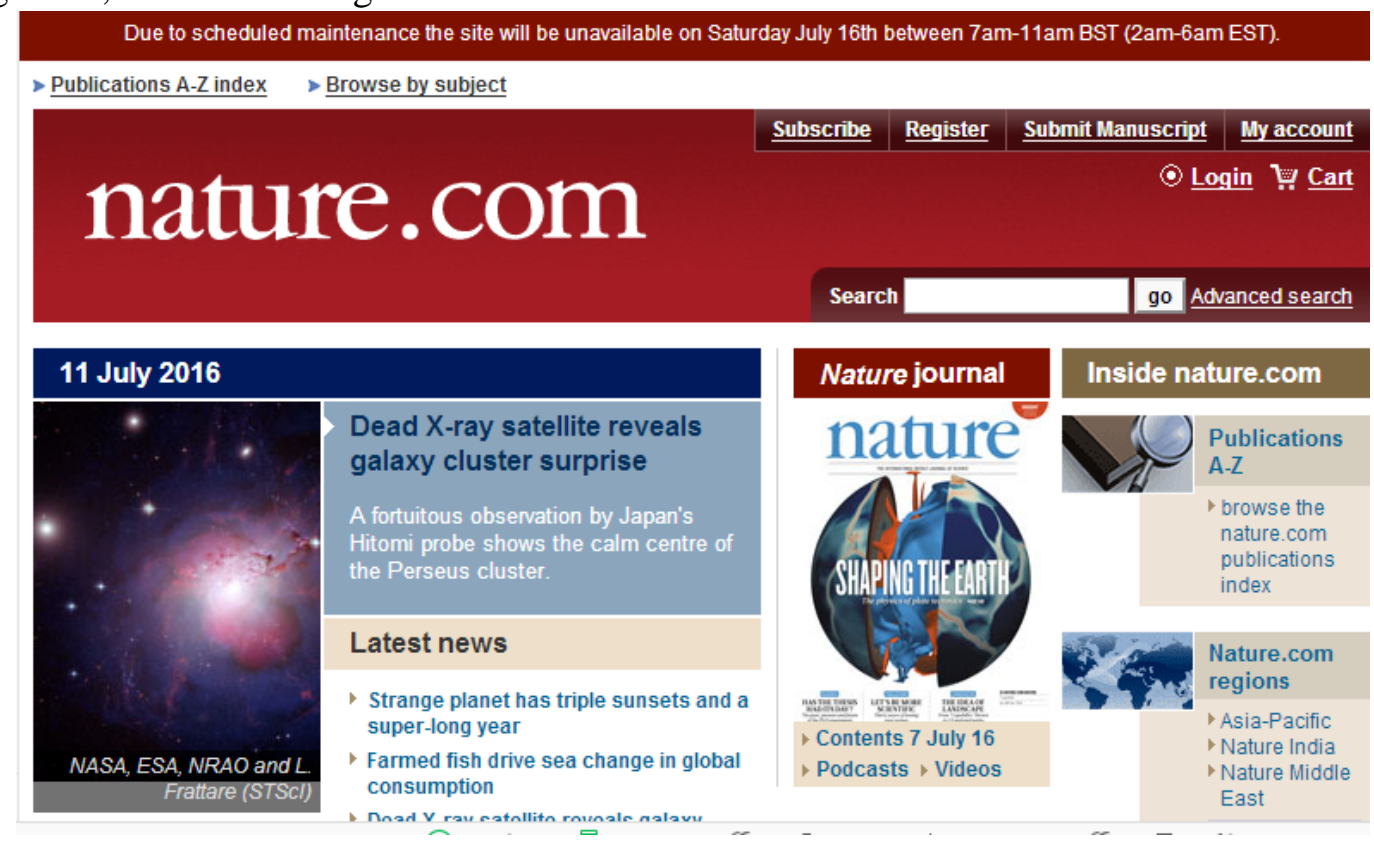

Figure 1. The appeal of different font, font size from:http://www.nature.com/index.html The Design of the Color

Color as the first visual language, its role before visual image, therefore has a direct influence on the effects of visual information. In addition, the color perception also influenced by people's psychological, with people's emotions, feelings, image, symbolism and other subjective factors related to mental health. 


\section{The General Requirements of Color Design}

The mass-tone attune of the page to clear. Selected mass-tone attune, the theme of the page should be first grasped different themes of the mass-tone attune of the web page with the corresponding to meet people's visual psychology.

Color collocation to reasonable and appropriate . Some visitor is more focused on the richness of color, the degree of change, and some browsers will fully consider color and other visual elements of the overall relationship. As a result, the page graphics and color collocation, the color collocation of the graphics and text, the graphics and text overall tone of harmonious and unified to attract attention.

Rich colors, bright, but moderation, prevents to produce visual fatigue. If the page is too colorful, easy to make them too much attention to local page, resulting in browsers ignore the key content; and the color is too bright-colored, contrast is too strong, easy to cause the visitor's visual fatigue, vision and even damage your visitors.

\section{Color Design Method}

(1)The use of monochrome pages. Website design should avoid using a single colour, lest produce drab feeling, But by adjusting the color saturation and transparency can also make the page changes, so that the site to avoid monotony. Which is a special kind of black colour, if used properly, reasonable design, often have very strong artistic effect.

(2) The use of contrasting colors in pages . Contrasting colors can produce strong visual result, through the rational use of contrast can make website characteristic, the highlight. Generally when the design is given priority to with a color hue, contrast color as an ornament, can have the effect that make the finishing point. Priceton University of Pennsylvania web page is shown in Figure 2 uses the strong contrast of changes in temperature very strong visual impact.

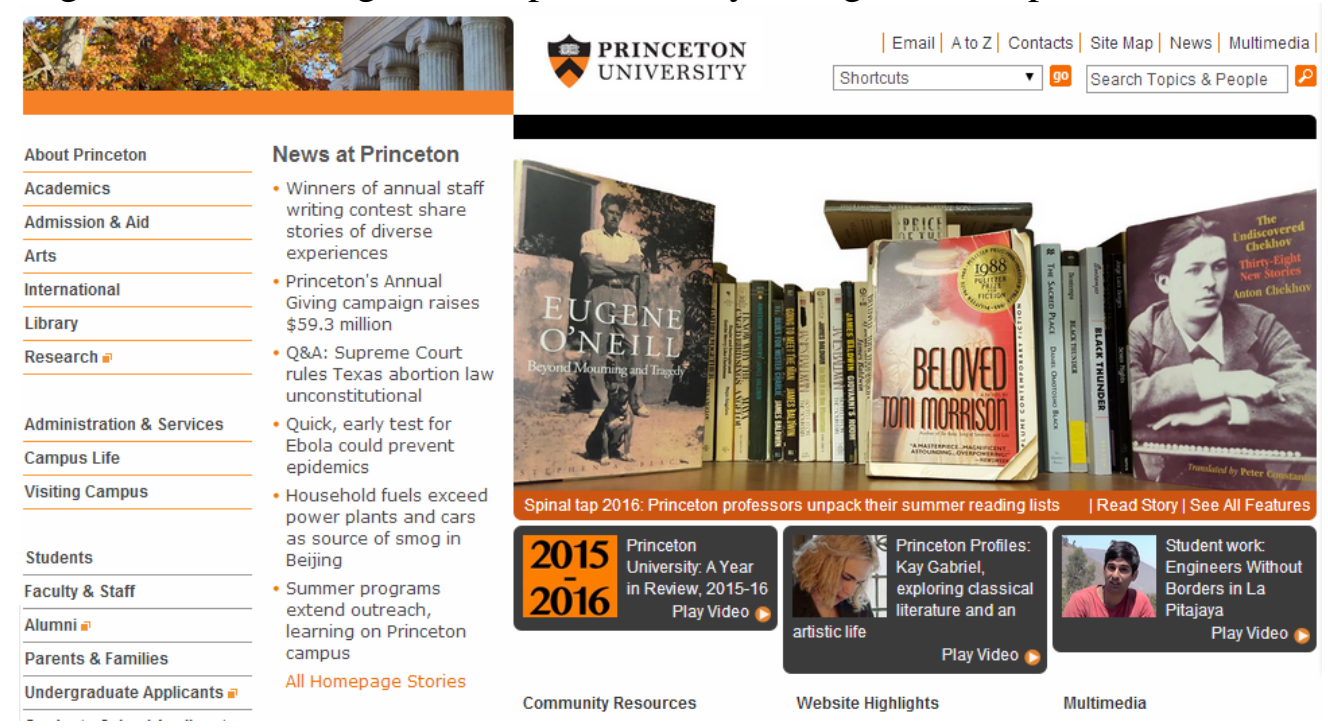

Figure 2 color contrast. From: http://www.princeton.edu/main/

\section{Design of Graphic computer vision}

Graphics is a feature of the website; it has the function of the smart, attractive, and conveys information. Good, moderate and clear graphic can make the page grace, the opposite to them. otherwise it will be attracted Viewer antipathy. Closely integrated with the theme of graphics can provide rich contextual information, graphics and related text location near that can quickly search relevant to the correct character, so as not to increase your reading difficulties. Stanford University web page as shown in Figure 3, Each paragraph and related graphics arranged in parallel, and dotted line between each part apart, so convenient for visitors, and beautifies the page.

Appropriate use of graphics can attract the attention of visitors, to help your visitors breakthrough the difficulties of abstract thinking, better understanding of the content, But not so in the page is inserted into the graph, so easy to make the page is too loud, and easy to disperse the 
visitor's attention, and even make them to produce visual fatigue, is not conducive to the effective information communication.

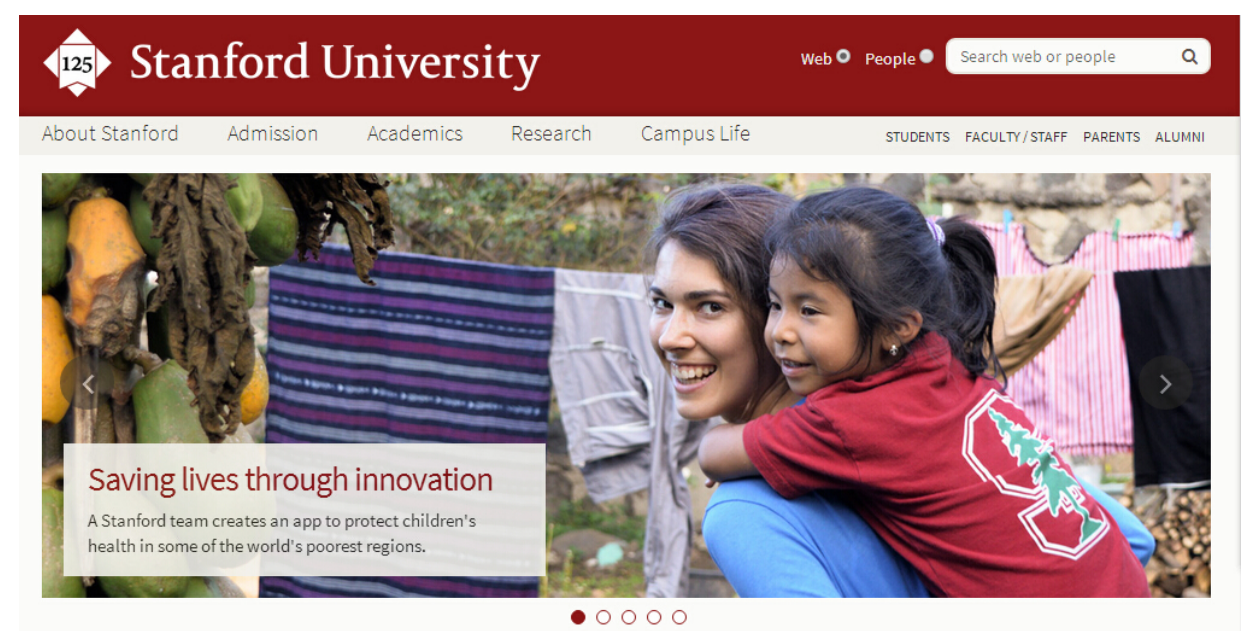

Figure 3 words and related graphic arrangement.From:http://www.stanford.edu/

\section{Format Layout Design of computer vision}

Fundamental purpose of computer vision design is for the sake of effective dissemination of information; page layout should be carried out according to the needs of specific expression content to creative ideas. As a designer, not only to convey the visual perception of beauty and the information content of a site to your visitors, more important is widely mobilizing the visitor's interest.

Using tables and framework for page segmentation technology, can make the theme clear, distinct, is planning page space and an important means to avoid clutter. At the time of the partitioning proportion should be paid attention to page space, the actual condition and the change of direction and color.

The purpose of designers carefully designed web page is in order to attract the attention of visitors, keep the visitor's eyes, Web pages without content is of no practical significance, information is the core of the site, is the basis of web design. But when the page layout, if the entire page layout is compact, all leave a bit gap, it will make person feel very dense opaque no breathing space, On the contrary, the appropriate space design makes the page layout has elastic and brings good daydream space, show some vitality. Stanford University web page as shown in Figure 4, the text of the compact layout and the overall density contrast to large area of white space, Web design is concise, no redundant adornment, give person with pure and fresh feeling.

\section{Stanford $\mid$ ENGINEERING}

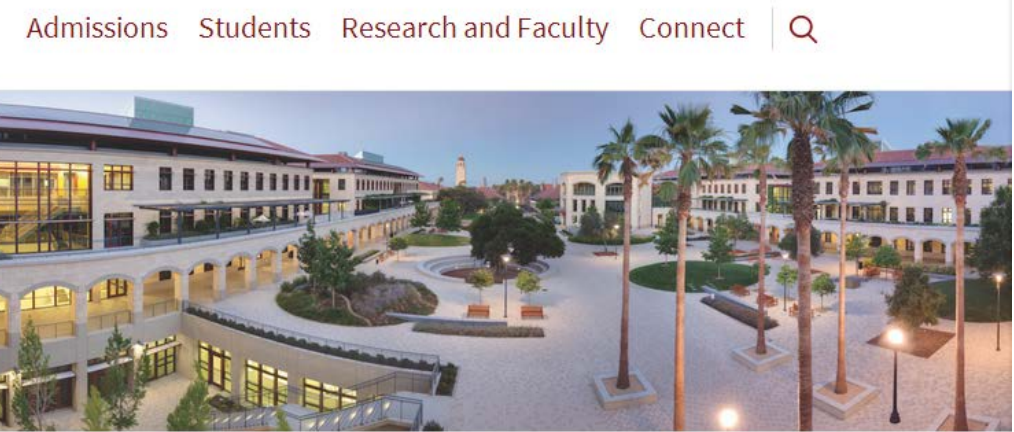

Figure4.font layout and page blank density contrast. From:https://engineering.stanford.edu/ 


\section{Summary}

At present, the study of teaching websites are mainly concentrated in the aspect of teaching design and technology to solve, to the humanized design of the page is not enough attention. From the computer vision design theory as a guide, combined with the specific teaching content, resulting in creative design and intuitive demonstrated, it makes teaching the content of the website has rich connotations, also has high aesthetic value. In this paper, from the Angle of computer vision, elaborates the teaching website design and analysis An example was given and the theory of computer vision design and penetration into the idea of teaching website design, Aims to fundamentally change the people in the past the one-sided understanding of teaching web site design, architecture is a comprehensive, integrated teaching website design concept.

\section{Acknowledgments}

The work was supported by the funding plan for the young backbone teachers of higher education institutions in Henan Province. The work was supported by the Post Doctoral Orientation Funding Project of Yunnan Province in 2015. The work was supported by the Project of Youth Science Foundation of National Natural Science Foundation with the project number 41505015 and the project name Measurement Method of Atmospheric High-resolution Microwave Radiometric Based on Virtual Mirror Antenna. The work was also supported by the scientific research project of Wuhan Technology and Business University with the project number A2015005 and the project name Non-destructive Testing System of Porous Ceramic Structure Based on Multi-frequency Eddy Current and Machine Vision.

\section{References}

[1] X. Q. Zhang, J. Q. Gu, W. Cao, J. G. Han, Akhlesh Lakhtakia, W. L. Zhang, Bilayer-fish-scale ultrabroad terahertz bandpass filter, OPTICS LETTERS 37: 906-908(2012).

[2] J. G. Han, J. Q. Gu, X. C. Lu, M. X. He, Q. R. Xing, W. L. Zhang, Broadband resonant terahertz transmission in a composite metal-dielectric structure, OPTICS EXPRESS 17 : 16527-16534(2009). [3] D. R. Smith, D. C. Vier, T. Koschny, C. M. Soukoulis, Electromagnetic parameter retrieval from inhomogeneous metamaterials, Phys.Rev. E, 71, 036617-036620(2005).

[4] A. Mary, S. G. Rodrigo, F. J. Garcia-Vidal, L. Martin-Moreno, Theory of negative-refractiveindex response of double-fishnet structures, Phys. Rev. Lett. 101, 103902- 10305 (2008).

[5] Th. Koschny, P. Markos, E. N. Economou, D. R. Smith, D. C. Vier, C. M. Soukoulis, Impact of inherent periodic structure on effective medium description of left-handed and related metamaterials. Phys. Rev. B, 71, 245105-245127 (2005).

[6] A. F. Starr, P. M. Rye, D. R. Smith, S. Nemat-Nasser, Fabrication and characterization of a negative-refractive-index composite metamaterial. Phys. Rev. B 70, 113102-113105 (2004).

[7] C. Caloz, T. Itoh, Electromagnetic Metamaterials: Transmission Line Theory and Microwave Applications (John Wiley-IEEE Press, 2005).

[8] G. V. Eleftheriades, K. G. Balmain, Negative Refraction Metamaterials: Fundamental Principles and Applications (Wiley-IEEE Press, 2005).

[9] J. G. Han, J. P. Gu, X. H. Lu, M. X. He, Q. R. Xing, W. L. Zhang, Broadband resonant terahertz transmission in a composite metal-dielectric structure, OPTICS EXPRESS, 17:16524-16527(2009)

[10] M. Zhong, Y. H. Ye, Role of coupling of localized surface plasmon modes in transmission properties of compound structure metamaterials, Optics Communications, 335:94-98(2015)

[11] J. Yang, C. Sauvan, H. T. Liu, P. Lalanne, Theory of fishnet negative-index optical metamaterials, Phys. Rev. Lett, 107:043903

-043906 (2008). 